5. HISTORIA DEL DERECHO DE AMÉRICA 

Revista de Estudios Histórico-Jurídicos [Sección Historia del Derecho de América] XXXII (Valparaíso, Chile, 2010) [pp. 383 - 394]

\title{
LA TRADICIÓN ROMANÍSTICA EN LOS ESTADOS UNIDOS CON UNA NOTA SOBRE EL ESTADO DE LA FLORIDA
}

[Romanistic Tradition in the United States with a Note Regarding the State of Florida]

\author{
Matthew C. Mirow \\ Universidad Internacional de Florida, Miami*
}

\section{RESUMEN}

El artículo examina la presencia del Derecho romano en la doctrina y jurisprudencia de los tribunales de Justicia de los Estados Unidos de Norteamérica, con especial referencia al Estado de Florida.

Palabras clave: Civil Law en USA. Derecho romano en USA.

\section{AbSTRACT}

This article examines the presence of Roman Law in the doctrine and jurisprudence of the Courts of Justice of the United States of America, with especial reference to the State of Florida.

Keywords: Civil Law in USA. - Roman law in the USA.

Este trabajo hace algunas observaciones sobre el uso del Derecho romano en la Historia del Derecho de los Estados Unidos basado en algunos estudios recientes publicados en dicho país. No voy a tratar de hacer una presentación completa de toda la literatura sobre el tema, pero sólo quiero usar algunos artículos recientes para dar unos ejemplos que muestran el desarrollo del tema. Con estos estudios en mente, quiero hacer unos comentarios sobre la manera en que el Derecho romano se usó frente a las Cortes del Estado de la Florida.

Nadie duda que el Derecho de los EE.UU. y el Derecho de los Estados de los EE. UU. es del "common law" conocido como el Derecho común, el Derecho

* Profesor de Derecho de la Universidad Internacional de Florida, Miami, Florida, EE.UU.; Profesor visitante de la Pontificia Universidad Católica de Valparaíso; Fulbright Scholar, Chile. Dirección postal. Correo electrónico: mirowm@fiu.edu El autor agradece el Dr. D. Patricio Carvajal y la Schola Serviana Iuris Romani. 
anglosajón, o el Derecho anglo-americano. Sin embargo y por supuesto, hubo influencias de otros sistemas del Derecho en su breve historia. Podemos decir que el Derecho romano ha sido una de las fuentes pequeñas pero importantes en la historia del Derecho de los EE.UU. Aquí, voy a hablar sobre el uso del Derecho romano durante la época colonial inglesa y las primeras décadas de la república y el uso del Derecho romano por las élites intelectuales en el siglo XIX. Desde aquí, podemos considerar el uso del Derecho romano por la Corte Suprema de los Estados Unidos, y el uso del Derecho romano en los tribunales de los Estados. También, voy a añadir algunas observaciones sobre el uso del Derecho romano en los tribunales de la Florida.

\section{El Derecho colonial de los Estados Unidos Y EN LAS PRIMERAS DÉCADAS DE LA REPÚBLICA}

Existen muy pocos estudios sobre el uso del Derecho romano en la época colonial inglesa. Según los estudios de Hamilton Bryson sobre el Estado de Virginia, una de las culturas jurídicas más elevadas en esos tiempos, las bibliotecas de Virginia tenían más de 25 tomos sobre Derecho romano y Derecho civil incluyendo el Corpus iuris civilis entero, con las Institutiones, por cierto, los comentarios de Vinnius y Heineccius, y las obras de Jean Domat, entre otros ${ }^{1}$.

Los reportajes de los casos de la Virginia colonial muestran el uso del Derecho romano de vez en cuando. Por ejemplo, según Bryson, entre 1733 y 1743 el fiscal general de la colonia, Edward Barradell, usaba las obras de Jean Domat y mencionaba el Derecho romano frecuentemente en sus argumentos ${ }^{2}$. Esto no es mucha evidencia, pero sabemos que al menos una colonia tenía obras sobre el Derecho romano y que los abogados las usaban como fuente.

En el Estado de Virginia, el uso del Derecho romano continuó como fuente supletoria en las últimas décadas del siglo xviii, sobre todo con el gran jurista de Virginia, George Wythe, canciller de un tribunal importante del Estado y profesor de Derecho en el College of William and Mary. El usó las Institutiones, los Digesta y el Codex varias veces en sus sentencias. Bryson nota que también el Derecho romano se usaba mientras los abogados hablaban de la distribución de los bienes de difuntos y en casos sobre el almirantazgo ${ }^{3}$.

Según Bryson, la cima del Derecho romano en Virginia fue entre 1776 y 1830 y George Wythe tuvo un papel importante en su uso. Después de esta fecha, hubo suficientes precedentes de Virginia y los abogados ya no tenían que buscar soluciones en fuentes ajenas como el Derecho romano ${ }^{4}$.

\footnotetext{
${ }^{1}$ Bryson, W. Hamilton, The Use of Roman Law in Virginia Courts, en Ratio Decidendi: The Guiding Principles of Judicial Decisions, Part II: Foreign Law (Serge DAUCHY, W. Hamilton Bryson, and M.C. Mirow, ed., Berlin, forthcoming 2010).

${ }^{2}$ Ibíd.

${ }^{3}$ Ibíd.

${ }^{4}$ Ibíd.
} 


\section{El Siglo XIX: LA ALTA CULTURA JURÍDiCA EN LOS Estados UnidOS}

Según los historiadores del Derecho Michael Hoeflich y Peter Stein, el Derecho romano tuvo un papel importante para los juristas y académicos legales en el mundo anglo-americano desde fines del siglo XVIII hasta comienzos del siglo XX. Esto es verdad sobre todo en lo que se llamaba la alta cultura jurídica de los profesores de Derecho del siglo XIX dentro de las facultades elitistas de los Estados Unidos.

En este aspecto, se destaca la primera mitad del siglo XIX en los Estados Unidos. Según Hoeflich hubo algunos círculos o células del Derecho romano en ciudades importantes de esa época. Hubo un grupo en Boston que incluyó a Joseph Story, magistrado de la Corte Suprema de los EE.UU. entre 1811 y 1845 y Simon Greenleaf, profesor de Derecho en Harvard en la primera mitad del siglo XIX. Para ellos, el estudio del Derecho romano fue parte de un proyecto más grande de estudiar la historia y la sociedad para ser buenos seres humanos y buenos abogados. Leyeron casi todo, pero el Derecho romano, como un sistema complejo y pragmático de una cultura y sociedad respetada, fue particularmente importante para estos abogados. También, según Hoeflich, el estudio del Derecho romano fue una manera de diferenciar la educación "científica" legal de la universidad frente a la manera usual de esa época, un aprendizaje dentro de la oficina de un abogado mayor ${ }^{6}$.

Durante la misma época hubo un grupo de romanistas en el Estado de Nueva York. Un juez famoso, el canciller James Kent, fue uno de los más destacados de este grupo. Ellos, según Hoeflich, tenían intereses más anticuarios e históricos. Por ejemplo Kent, frente al colegio de abogados de Nueva York en 1836 habló con entusiasmo sobre los civilistas del foro romano y dio loores a Cicerón y a sus obras $^{7}$. Es triste, pero verdad, según Alan Watson, que en las sentencias donde Kent actuaba como juez usaba el Derecho romano, no sabía, empero, como citarlo ni interpretarlo con la precisión de un buen romanista ${ }^{8}$.

Otros grupos se centraron en los Estados de Luisiana y de Carolina del Sur. Para ellos, el Derecho romano podía ser una fuente importante y directa en la reforma del Derecho substantivo del Estado. Aquí, Hoeflich nota la influencia de abogados de Luisiana y la tradición de las familias grandes y ricas de esta región, de hacer sus estudios posgraduados en Escocia o en Europa?

${ }^{5}$ Astorino, Samuel J., Roman Law in American Law: Twentieth Century Cases of the Supreme Court, en Duquesne Law Review, 40 (2001-2002), p. 632; Hoeflich, Michael J., Roman and Civil Law and the Development of Anglo-American Jurisprudence in the Nineteenth Century (Athens, Georgia, 1997), p. 142; STeIn, Peter, The Attraction of the Civil Law in Post-Revolutionary America, en Virginia Law Review, 52 (1966), p. 403.

${ }^{6}$ Hoeflich, Michael H., Roman Law in American Legal Culture, en Tulane Law Review, 66 (1992), pp. 1.725-1.732.

${ }^{7}$ Ibíd., pp. 1.732-1.734.

${ }^{8}$ Ibíd., p. 1.735, con cita de Watson, Alan, Chancellor Kent's Use of Foreign Law, en Reimann, Matthias A. (editor), The Reception of Continental Ideas in the Common Law World, 1820-1920 (Berlin, 1993).

${ }^{9}$ Hoeflich, Roman Law in American Legal Culture, cit. (n. 6), pp. 1.735-1.738. 
¿Qué tenían estos grupos en común? Primero cada grupo tenía unos individuos que habían estudiado el Derecho más allá de lo normal, es decir, habían tenido un aprendizaje con otro abogado. Segundo, cada grupo tenía algunos lazos con Europa y con el Derecho continental, usualmente en Alemania o en Escocia. Tercero, cada grupo estaba vinculado con una revista de literatura o de Derecho en que publicaban, de vez en cuando, sus investigaciones ${ }^{10}$. Finalmente, cada grupo también tenía acceso a una biblioteca -privada o universitaria- con suficientes fuentes de Derecho romano ${ }^{11}$.

En pocas palabras, ¿qué podemos decir sobre estas bibliotecas? Ellas contenían las Institutiones de Justiniano y los comentarios de Vinnius y las obras de Cicerón. De vez en cuando contenían el Corpus iuris entero. La biblioteca privada de Swinto Legaré, socio del grupo de Carolina del Sur, tenía más de 250 tomos en latín, francés, alemán e italiano y la mayor parte eran obras sobre Derecho romano y civil. Hubo traducciones importantes de fuentes romanas en los Estados Unidos en esta época, incluyendo una edición de las Institutiones publicada en 1812 por Thomas Cooper, también socio del grupo de Carolina del Sur ${ }^{12}$.

En otro estudio, Hoeflich describe como estos abogados consiguieron esos libros de Derecho romano y Derecho civil. Hubo tres maneras importantes: por medio de amigos en Europa (la amistad entre Joseph Story y el jurista alemán Mittermaier es bien conocida); por ser traídos de Luisiana, que siempre fue una fuente importante del Derecho romano y civil en los Estados Unidos; y por las librerías jurídicas que conocían el mercado y la demanda por estos libros ${ }^{13}$.

El uso del Derecho romano en los Estados Unidos por la alta cultura jurídica continuó por mucho más tiempo que el uso de éste en los tribunales tempranos de los nuevos Estados. Parece que la alta cultura jurídica se decepcionó con el Derecho romano alrededor de 1920. Podemos considerar dos razones para esto. Primero, durante y después de la Primera Guerra Mundial, hubo un sentimiento anti-alemán grande y la influencia de la cultura alemana en todos los aspectos de la vida intelectual -incluyendo su liderazgo en el campo del Derecho romano- bajó significativamente. Segundo, alrededor de esta década, la enseñanza de los idiomas griego y latín disminuyó en los Estados Unidos y los estudiantes de Derecho no tenían la capacitación para leer y estudiar los textos de Derecho romano ${ }^{14}$. Sin embargo, estos grupos de pensadores y juristas tuvieron una influencia grande en el desarrollo del Derecho en los Estados Unidos por sus trabajos como jueces y por sus tratados sobre el Derecho substantivo de los Estados Unidos.

\footnotetext{
${ }^{10}$ Ibíd., p. 1.726.

${ }^{11}$ Ibíd., p. 1.726.

${ }^{12}$ Ibíd., pp. 1.738-1.743.

${ }^{13}$ Hoeflich, Michael H., Bibligraphical Perspectives on Roman and Civil Law, en Law Library Journal, 89 (1997), pp. 48-53.

${ }^{14}$ Astorino, Roman Law in American Law, cit. (n. 5), p. 628.
} 


\section{UNA NOTA SOBRE LAS SENTENCIAS}

Antes de hablar sobre el Derecho romano en las sentencias de los tribunales, hay que hablar un poco sobre el contenido de las sentencias y su motivación.

Cuando encontramos una cita al Derecho romano en una sentencia de un tribunal de los Estados Unidos, tenemos que pensar en la intención del juez en poner o usar dicha fuente. En su estudio sobre la jurisprudencia del magistrado Edward Douglass White, Reid nota que una sentencia y su motivación dentro del sistema del Derecho anglo-americano tenía como objeto el de convencer a las partes, los abogados, y a otros jueces de la manera más persuasiva de que la decisión era la correcta. Según Reid, esto quiere decir que el juez buscaría con eficiencia la selección de sus fuentes con el fin de crear una opinión que tuviera el efecto de persuasión más poderoso posible ${ }^{15}$. Otros académicos han dicho que el uso del Derecho romano en las sentencias viene a ser nada más que un adorno intelectual, una manera de deslumbrar frente a los otros abogados y jueces. Creo que la evidencia muestra que el Derecho romano tenía un papel aún más importante.

\section{La Corte Suprema de los Estados Unidos}

En la jurisprudencia de la Corte Suprema de los Estados Unidos, el Derecho romano siempre ha tenido un papel supletorio en el pensamiento de la Corte. Charles Reid recientemente ha estudiado el uso del Derecho romano y canónico por el presidente, magistrado Edward Douglass White, en sus sentencias en la Corte Suprema de los Estados Unidos entre la década de los 90 del siglo XIX hasta la década de los 20 del siglo XX. Este estudio da información fundamental y básica sobre un debate que existe hoy en día sobre el uso del Derecho extranjero en las sentencias de la Corte Suprema de los Estados Unidos. Y este debate también forma parte de un debate aún más grande sobre lo que se llama el excepcionalismo de los Estados Unidos y su sistema legal ${ }^{16}$.

En este contexto, Edward White fue inusual como magistrado de la Corte Suprema porque él fue abogado del Estado de Luisiana y tuvo una educación legal basada en la tradición romana civilista. Reid descubre que White usó esta educación en su trabajo en la Corte y el uso del Derecho romano fue aceptado, de vez en cuando, por los otros magistrados ${ }^{17}$.

Uno de los casos analizado por Reid es un caso de Derecho penal de 1894, "Coffin v. United States", que establece la regla de la presunción de inocencia ("presumption of innocence") de un acusado en el Derecho de los EE.UU. White trata de mostrar la antigüedad y la universalidad de la idea con el uso de fuentes del Derecho romano. White usó cinco fuentes del Derecho romano incluyendo

\footnotetext{
${ }^{15}$ ReID, Charles J., Jr., Edward Douglass White's Use of Roman and Canon Law: A Study in the Supreme Court's Use of Foreign Legal Citation, en University of Saint Thomas Law Journal, 3 (2005), pp. 284-285.

${ }^{16}$ Ibíd., pp. 281-284.

${ }^{17}$ Ibíd., p. 284.
} 
un decreto conjunto del año 382 por Graciano, Valentiniano II y Teodosio que requirió testigos suficientes para apoyar dicha acusación y unos pasajes de los Digesta de Justiniano sobre la interpretación de leyes en causas criminales o cuando hay dudas sobre el texto. White también mencionó el dicho famoso del emperador Trajano que dice "es mejor que un crimen quede sin castigo a que una persona inocente sea castigada". Una fuente adicional fue un pasaje del Derecho canónico del Liber Extra de Gregorio IX sobre la calidad de evidencia en juicios de la herejía. Reid leyó correctamente estas fuentes para concluir que ninguno apoya directamente la idea de la presunción de inocencia del acusado ${ }^{18}$.

Otra fuente que White usó era romana, pero no legal. White usó la obra de Ammianus Marcellinus del siglo IV sobre la vida del emperador Juliano, quien se hizo César en 359. En el contexto de acusaciones contra Numerius, anteriormente gobernador en Galia, Juliano respondió, "Ecquis ait innocens esse poterit, si accusasse sufficiet?” (“¿Existe alguien que sea inocente si para ser culpable sólo es suficiente que otro te haya acusado?"). Aquí tenemos la oración del mundo romano que suena como la presunción de la inocencia. Aunque las fuentes del Derecho romano no establecen la presunción de inocencia en el mundo romano, las fuentes ayudaron en el proceso de hablar sobre la antigüedad y la universalidad de la idea. De hecho, Reid ha mostrado que, tan recientemente como 1987, el magistrado Thurgood Marshall ha citado el pasaje de Ammianus Marcellinus mientras hablaba sobre la presunción de inocencia ${ }^{19}$.

Vale la pena mencionar que para muchos abogados norteamericanos del siglo XIX, el Derecho romano tenía un espectro bastante amplio. Es decir, que el Derecho romano no solo significaba el Corpus iuris sino también las obras de Cicerón y Quintiliano como ejemplos de buenos oratorios y fuentes de ideas básicas sobre el Derecho. No debemos olvidar que las muestras de buena oratoria fueron muy importantes en el Derecho común debido a que la habilidad oral en los juicios y frente a los jurados era muy útil ${ }^{20}$.

Samuel Astorino, profesor en Pennsylvania, recientemente ha escrito sobre el uso del Derecho romano por la Corte Suprema de los Estado Unidos en el siglo XX. Después de leer y de analizar aproximadamente veinticinco sentencias en que la Corte menciona el Derecho romano, Astorino concluye que el uso del Derecho romano por la Corte es generalmente encontrado dentro del contexto de una presentación histórica de una regla o una norma particular. Las citas al Derecho romano son frecuentemente acompañadas por referencias a materias como el Code Napoléon, las Siete Partidas y los tratados de civilistas ${ }^{21}$.

\footnotetext{
${ }^{18}$ Ibíd., pp. 286-288.

${ }^{19}$ Ibíd., pp. 288-291.

${ }^{20}$ Clark, Carolyn -. Hoeflich, M.H., Roman Law as "ratio decidendi" in Early American Law, en Ratio Decidendi: The Guiding Principles of Judicial Decisions, Part II: Foreign Law (Serge Dauchy, W. Hamilton Bryson, and M.C. Mirow, ed., Berlin, forthcoming 2010).

${ }^{21}$ Astorino, Roman Law in American Law, cit. (n. 5), p. 627.
} 


\section{Tribunales estatales}

Los estudios de Michael Hoeflich nos han enseñado que es demasiado fácil exagerar el papel del Derecho romano, aparte del Estado de Luisiana, en el desarrollo de normas sustantivas en el Derecho de los Estados. Debo mencionar que Luisiana esta repleta de casos que usan el Derecho romano y el Derecho civil europeo y por eso está excluida de esta presentación. También, el Derecho romano tuvo una influencia grande sobre el Derecho de las aguas en Texas y sus Estados vecinos ${ }^{22}$.

Al considerar los argumentos de Stein y Hoeflich de que el Derecho romano tuvo un efecto mucho más importante en la alta cultura jurídica y no tanto con los abogados ordinarios o dentro de los tribunales, Richard Helmholz, profesor de la Universidad de Chicago, decidió leer todos los casos entre 1790 y 1825 y buscar el uso del Derecho romano en las cortes. Consideró las sentencias de catorce Estados con reportajes de suficientes casos para estudiar ${ }^{23}$. Según Helmholz: "Entre 1790 y 1825 , la variedad de fuentes citadas por los abogados norteamericanos fue considerablemente grande. Los textos básicos del Derecho romano -Institutas, Código, Digesto, y Novelas- fueron frecuentemente citados y comentados" ${ }^{24}$.

En algunos campos del Derecho en los casos de esta época se destacaba el uso del Derecho romano incluyendo el Derecho marítimo y el Derecho comercial internacional. Con la excepción de Carolina del Sur, donde ya había un grupo de romanistas, el Derecho romano no fue usado en casos sobre la esclavitud. Según Helmholz, esto es una cosa rara cuando uno considera la abundancia de reglas y normas que existían sobre la esclavitud en el Derecho romano ${ }^{25}$.

¿De qué manera servía el Derecho romano en estos casos? Según Helmholz, son tres las razones. Primero, para llenar huecos donde no había regla o norma del Derecho común. Segundo, para hablar de derechos fundamentales extraídos del Derecho natural y el ius gentium hasta proveer argumentos para anular leyes de las legislaturas de los Estados. Tercero, como adorno, para apoyar una regla de Derecho común, o cuando el Derecho civil era parte del Derecho común, o para mostrar que una idea era universal entre muchos sistemas del Derecho ${ }^{26}$.

Con las obras de Helmholz y otros, podemos decir que el Derecho romano no fue exclusivamente el campo de pocos hombres elitistas de la alta cultura jurídica de la primera mitad del siglo XIX, sino que tambien tenía un uso usual dentro de los tribunales de esa época, pero sin embargo fue un uso estadisticamente bastante pequeño.

En resumen, ¿que observaciones generales podemos hacer?

a) En la época colonial y durante las primeras décadas de los nuevos Estados de

${ }^{22}$ BaAde, Hans W., Roman Law in the Water, Mineral and Public Land Law of the Southwestern United States, en The American Journal of Comparative Law, 40 (1992), pp. 865-877.

${ }^{23}$ Helmholz, R. H., Use of the Civil Law in Post-Revolutionary American Jurisprudence, en Tulane Law Review, 66 (1992), pp. 1649-1652.

${ }^{24}$ Ibíd., p. 1.654 .

${ }^{25}$ Ibíd., p. 1.657-1.664.

${ }^{26}$ Ibíd., p. 1.664-1.682. 
la nueva república, el Derecho romano tenía un papel supletorio significativo en el ejercicio de la abogacia y en las decisiones de los tribunales. El uso del Derecho romano en los tribunales disminuyó bastante como fuente supletoria después de 1830 cuando ya hubo suficientes precedentes norteamericanos.

b) En la primera mitad del siglo XIX, el Derecho romano fue una fuente importante usada por un élite legal en los Estados Unidos. Estos pensadores del Derecho y juristas usaron el Derecho romano, según Hoeflich, para estructurar el Derecho, para proveer una terminología sobre importantes ideas jurídicas, $y$ para comparar el Derecho común. El interés en el Derecho romano a nivel elitista disminuyó durante la Primera Guerra Mundial y la falta de educación en latín de los estudiantes de Derecho contribuyó a la caída de dicho interés.

c) Esta tradición fue reflejada en la jurisprudencia de la Corte Suprema de los Estados Unidos cuando uno de los magistrados venía de uno de estos grupos, como Joseph Story u Oliver Wendell Holmes. También, en el caso del magistrado Edward Douglass White, su experiencia con el Derecho de Luisiana tuvo efecto en su jurisprudencia.

d) Aunque las épocas de oro del Derecho romano en los Estados Unidos ya habían pasado, uno como profesor o abogado sigue viendo citas y referencias al Derecho romano de vez en cuando en sentencias de los tribunales federales y estatales hasta hoy en día. Generalmente, estas referencias forman parte de una presentación histórica de una idea o regla y no son ofrecidas como alternativa al Derecho común.

\section{Las Sentencias de los tribunales del Estado de la Florida.}

Juan Ponce de León llegó a la Florida en 1513 e hizo otra exploración de la tierra en 1521. Hernando de Soto también pasó por la Florida en 1539. En 1565, Pedro Menéndez fundó la ciudad de San Agustín, la primera población europea en lo que es hoy los EE.UU. Aunque hubo ataques franceses e ingleses por décadas, la región permaneció bajo control español hasta 1763, cuando pasó a los ingleses por un tratado con España. Florida fue leal a la corona inglesa durante la guerra de independencia, pero fue capturada de nuevo por los españoles con ayuda de los franceses en 1784. Aunque bajo el control español, la región recibió muchos inmigrantes del norte y por lo tanto se convirtió en una región que tenía más vínculos con la cultura inglesa que las provincias españolas del sur. La Florida se integró a los EE.UU. como territorio en 1821, por el Tratado Adams-Onís. Entre 1835 y 1842 , el territorio fue sitio de una guerra fuerte entre los soldados de los EE.UU. y la tribu Seminole. El territorio se hizo Estado de los EE.UU. en $1845^{27}$.

La Florida nunca tuvo la oportunidad de compartir las épocas más importantes del uso del Derecho romano así como lo tuvieron otras partes de los EE.UU. Hasta 1830, durante la primera época, no tenía tribunales propios como Estado.

\footnotetext{
${ }^{27}$ Florida Division of Historical Resources, http://www.fheritage.com/facts/history/ summary/.
} 
Tampoco, durante el siglo XIX, tenía un centro cultural o legal para desarrollar un comunidad de romanistas como sí lo tenían Boston o Carolina del Sur. En la fecha en que se hizo Estado, ya la ola de auge más grande del Derecho romano en el país había pasado.

Sin embargo, a partir de 1845 se han encontrado más de 40 sentencias de los tribunales estatales que mencionan el Derecho romano y el Código Justiniano. ¿Qué podemos decir sobre estas sentencias? La gran mayoría de ellas mencionan el Derecho romano como una fuente supletoria y era consistente con el Derecho común.

También, entre estos casos, es frecuente que el Derecho romano esté incorporado en la sentencia por una cita o un tratado o un juez famoso que menciona el Derecho romano. Por ejemplo, el Derecho romano entra al Derecho del Estado de Florida en 1856 en un caso en que el juez cita a Joseph Story sobre la validez de contratos ajenos en el Estado ${ }^{28}$. Hay otros ejemplos en que pasajes de las obras de Story o Greenleaf, los dos del grupo de Boston, mencionan el Derecho romano y son citados por un juez en la Florida ${ }^{29}$. También, uno ve que se usa el Derecho romano para mostrar que una regla es consistente con la justicia natural en todos los sistemas del Derecho ${ }^{30}$.

Hay otros grupos de casos en que el juez quería distinguir el Derecho común del Derecho romano. Por ejemplo, en 1879 un juez de Florida cita las obras de Story para demostrar que los remedios usados en el Derecho romano para hacer una contabilidad dentro de una comanditaria eran mejores que los remedios usados en el Derecho común ${ }^{31}$. En 1847, la Corte usa el Derecho romano para hacer una distinción entre una apelación (del Derecho romano-civil) y una orden judicial de revisión ("writ of error" del Derecho común) en los procedimientos civiles ${ }^{32}$. En 1882, la Corte nota que aunque un fiduciario de un fideicomiso no recibía remuneración bajo el Derecho romano, el "trustee” tenía el Derecho de recibir un pago por sus esfuerzos ${ }^{33}$. También, en 1926, la Corte nota la distinción entre el divorcio bajo el Derecho romano y el divorcio en los Estados de los EE.UU ${ }^{34}$.

Como Hoeflich nos ha dicho, de vez en cuando, es la precisión o la eficiencia o el nivel del desarrollo del Derecho romano el que guía a los abogados o jueces a utilizarlo. Hay ejemplos en que una palabra recibe una definición precisa por el Derecho romano en la jurisprudencia del Estado de la Florida. Aquí, quiero hablar de tres ejemplos: aluvión ("aluvium”), lucri causa y domicilio.

Primero, un caso de 1957 en que la Corte usa el Derecho romano para definir

28 “Crowell v. Skipper”, 6 Fla. 580 (1856).

29 "Randall v. Parramore \& Smith”, 1 Fla. 409 citado en "Battle v. Jennings Naval Stores Co.”, 74 Fla. 12, 75 So. 949 (1917); "Hyer v. Caro”, 17 Fla. 332 (1879); “In re Silverman's Estate", 163 So.2d 321 (1964).

30 "Holbrook v. Allen”, 4 Fla. 87 (1851); “Geiger v. Filor”, 8 Fla. 325 (1859); “Sullivan v. Richardson”, 33 Fla. 1 (1894); "Gray v. Gray”, 91 Fla. 103 (1926); “Cragin v. Ocean \& lake Realty” Co., 101 Fla. 103 (1926).

31 "Hyer v. Caro", 17 Fla. 332 (1879).

32 "Fraser v. Livingston", 1 Fla. 392 (1847).

33 “Muscogee Lumber Co. v. Hyer”, 18 Fla. 698 (1882).

34 “Herron v. Passailaique”, 92 Fla. 815 (1926). 
las fronteras de una parcela de bienes raices. En 1833, el gobierno de los Estados Unidos hizo una medición de un lote de 623 acres que bordeaba al Golfo de México y se llamó sección 15, aunque por costumbre una sección debería tener 640 acres. En 1856 el gobierno de los EE.UU. transfirió esa tierra al Estado de Florida. Entre 1865 y 1869, nuevas tierras lentamente se formaron al lado del Golfo de México, pero nunca fueron parte de la medición original. En 1883, el Estado de Florida transfirió las tierras a una empresa. La cuestión presentada a la Corte fue si la empresa era dueña de 623, 640 o de toda la tierra. La Corte empezó su sentencia con la distinción entre aluvión y avulsión en el Derecho romano. Tenemos aquí citas a las Institutiones de Gayo y a las Institutiones de Justiniano. La Corte nota que esta también fue la regla en Inglaterra y cita la regla desde los comentarios de Blackstone. También, esta es la regla usada por la Corte Suprema de los Estados Unidos y la regla aplicada anteriormente en la Florida. La transferencia entonces, incluyó las tierras obtenida por aluvión ${ }^{35}$.

En un segundo ejemplo, en 1921, la Corte Suprema de la Florida tenía que revisar una sentencia de culpabilidad en que el señor Maund autorizó al señor Groover a usar su carro para hacer unas diligencias vinculadas con los negocios de los dos. En cambio, el señor Groover llamó a su esposa y se fueron de vacaciones a los Estados del norte, Georgia y Carolina del Sur. El señor Groover dijó que iban a entregar el carro al Señor Maund después del viaje. Después de escuchar todos los hechos, el jurado en el tribunal de primera instancia decidió que el señor Groover ya tenía la intención de quedarse con el carro del señor Maund antes de recibirlo, y por lo tanto el señor Groover fue condenado por el crimen de hurto ${ }^{36}$.

Una de las preguntas presentadas a la Corte Suprema fue si hubo suficiente "lucri causa" como elemento necesario del hurto en el Estado de la Florida. Citando a Blackstone, la Corte dice que el "lucri causa" del Derecho romano es la misma cosa que el animus furandi del Derecho común y que según algunos casos del Estado de la Florida, la privación de la propiedad del dueño fue suficiente "lucrum" para satisfacer este elemento ${ }^{37}$.

El último ejemplo es sobre la definición de domicilio en el Estado de la Florida. En el caso de Smith contra Croom, un buque de vapor se hundió en 1837 yendo desde Nueva York hasta Charleston, Carolina del Sur. Hardy Croom, su esposa, y sus tres niños fallecieron en el accidente. Las leyes de sucesión de los dos Estados eran distintas y la Corte tenía que determinar el domicilio de los difuntos para distribuir sus bienes. La evidencia mostró que la familia quizás estaba en el proceso de trasladarse desde su quinta en Carolina del Norte hasta una nueva quinta en la Florida, pero nunca llegaron. En el proceso de determinar que el domicilio de Croom era Carolina del Norte, la Corte examina varias obras incluyendo las de Denizart, Pothier, y Story.

Sin embargo, es el libro de Phillimore sobre domicilio el que tuvo la influencia más grande ${ }^{38}$. El libro The Law of Domicile (1847) fue escrito por Sir

\footnotetext{
35 “Mexico Beach Corp. v St. Joe Paper Co.", 97 So.2d 708 (1957).

36 "Groover v. State", 82 Fla. 427; 90 So. 473 (1921).

37 “Groover v. State”, 82 Fla. 427, 431-432; 90 So. 473, 475 (1921).

38 "Smith v. Croom", 7 Fla. 81 (1857).
} 
Robert Phillimore un civilista inglés y socio de Doctors Commons en Londres. Phillimore usa y la Corte de la Florida cita el Derecho romano sobre domicilio para establecer una regla en que el domicilio es un sitio de donde uno no tiene la intención de salir ${ }^{39}$.

Desde el uso en este caso, dicha definición y su vínculo al Derecho romano continúan en la jurisprudencia del Estado de Florida hasta hoy en día. Desde 1857 uno encuentra al menos cinco sentencias que mencionan esta prueba para el domicilio y que también mencionan que viene del Derecho romano. Son casos de $1927,1955,1963,2000$, y tan recientemente como el año pasado ${ }^{40}$.

Así, podemos ver que el Derecho romano sigue teniendo una influencia en el Derecho de los Estados Unidos y también dentro de un Estado que no fue parte de la época de oro del Derecho romano en los Estados Unidos y que fue muy aislado de los grupos elitista del siglo XIX. En Florida, la influencia del Derecho romano no es muy grande, pero sí, allí está.

[Recibido el 30 de marzo y aprobado el 30 de julio de 2010].

BibLIOGRAFÍA

\section{Literatura}

Astorino, Samuel J., Roman Law in American Law: Twentieth Century Cases of the Supreme Court, en Duquesne Law Review, 40 (2001-2002).

Badde, Hans W., Roman Law in the Water, Mineral and Public Land Law of the Southwestern United States, en The American Journal of Comparative Law, 40 (1992).

Bryson, W. Hamilton, The Use of Roman Law in Virginia Courts, en Ratio Decidendi: The Guiding Principles of Judicial Decisions, Part II: Foreign Law (Serge DAuCHY, W. Hamilton Bryson, and M.C. Mirow, ed., Berlin, forthcoming 2010).

Clark, Carolyn - Hoeflich, M.H., Roman Law as "ratio decidendi" in Early American Law, en Ratio Decidendi: The Guiding Principles of Judicial Decisions, Part II: Foreign Law (Serge DAUCHY, W. Hamilton BrYsON, and M.C. Mirow, ed., Berlin, forthcoming 2010).

Helmholz, R. H., Use of the Civil Law in Post-Revolutionary American Jurisprudence, en Tulane Law Review, 66 (1992)

Heoflich, Michael H., Roman Law in American Legal Culture, en Tulane Law Review, 66 (1992).

Hoeflich, Michael H., Bibligraphical Perspectives on Roman and Civil Law, en Law Library Journal, 89 (1997).

Hoeflich, Michael J., Roman and Civil Law and the Development of Anglo-American Jurisprudence in the Nineteenth Century (Athens, Georgia, 1997).

ReID, Charles J., Jr., Edward Douglass White's Use of Roman and Canon Law: A Study

39 "Smith v. Croom", 7 Fla. 81 (1857).

40 "Wade v. Wade", 93 Fla. 1004 (1927); "Bloomfield v. City of St. Petersburg Beach", 82 So.2d 364 (1955); "In re Guardianship of Mickler", 152 So.2d 205 (1963); "Miller v. Gross", 788 So.2d 256 (2000); "Marshall v. Marshall”, 988 So.2d 644 (4th DCA 2008). 
in the Supreme Court's Use of Foreign Legal Citation, en University of Saint Thomas Law Journal, 3 (2005).

SteIn, Peter, The Attraction of the Civil Law in Post-Revolutionary America, en Virginia Law Review, 52 (1966).

Watson, Alan, Chancellor Kent's Use of Foreign Law, en Reimann, Matthias A. (editor), The Reception of Continental Ideas in the Common Law World, 1820-1920 (Berlin, 1993).

2. Casos

"Bloomfield v. City of St. Petersburg Beach", 82 So.2d 364 (1955).

“Cragin v. Ocean \& lake Realty” Co., 101 Fla. 103 (1926).

"Crowell v. Skipper", 6 Fla. 580 (1856).

"Fraser v. Livingston", 1 Fla. 392 (1847).

"Geiger v. Filor", 8 Fla. 325 (1859).

"Gray v. Gray", 91 Fla. 103 (1926).

"Groover v. State", 82 Fla. 427, 431-432; 90 So. 473, 475 (1921).

"Groover v. State", 82 Fla. 427; 90 So. 473 (1921).

"Herron v. Passailaique", 92 Fla. 815 (1926).

"Holbrook v. Allen", 4 Fla. 87 (1851).

"Hyer v. Caro", 17 Fla. 332 (1879).

"In re Guardianship of Mickler", 152 So.2d 205 (1963).

"In re Silverman's Estate", 163 So.2d 321 (1964).

"Marshall v. Marshall", 988 So.2d 644 (4th DCA 2008).

"Mexico Beach Corp. v St. Joe Paper Co.", 97 So.2d 708 (1957).

"Miller v. Gross", 788 So.2d 256 (2000).

"Muscogee Lumber Co. v. Hyer", 18 Fla. 698 (1882).

"Randall v. Parramore \& Smith", 1 Fla. 409, citado en "Battle v. Jennings Naval Stores Co.”, 74. Fla. 12, 75 So. 949 (1917).

"Smith v. Croom", 7 Fla. 81 (1857).

"Sullivan v. Richardson”, 33 Fla. 1 (1894).

"Wade v. Wade”, 93 Fla. 1004 (1927). 